\title{
Comportamiento del aborto séptico en pacientes que consultaron al Hospital San Vicente de Paúl. Universidad de Antioquia 1994-1997
}

\author{
Carlos Mena; José I. Gutiérrez; Wilson Múnera; Rubén Petro; Fabio Pineda*; Orlando Restrepo; William Botero**
}

\section{RESUMEN}

El aborto séptico constituye en la actualidad un problema médico, social y económico a nivel mundial debido a la gran demanda de casos, los elevados costos y las tasas de morbimortalidad materna. La Federación Internacional de Planificación Familiar calcula que cada año ocurren 5 millones de abortos inducidos; igualmente, en un estudio realizado en Colombia en 1990 se informaron 4.930 abortos en un período de 6 meses. El presente estudio es de tipo descriptivo retrospectivo, de corte transversal, llevado a cabo en el Departamento de Ginecología y Obstetricia del Hospital San Vicente de Paúl (HSVP) - Universidad de Antioquia, entre el 1º de octubre de 1997 y el 10 de marzo de 1998 ; en él se tuvieron en cuenta todas las pacientes que consultaron al HSVP entre el $1^{\circ}$ de enero de 1994 y el 31 de diciembre de 1997 . Se encontró que el grupo de edad más afectado fue el comprendido entre los 16-25 años, correspondiendo la mayor población a solteras y amas de casa. La gran nayoría de las pacientes eran multigestantes y la principal complicación fue el shock séptico.

PALABRAS CLAVES: Aborto séptico, gestantes, morbimortalidad, Hospital San Vicente de Paúl (HSVP).

\section{SUMMARY}

Septic abortion is actually one of the most important medical, social and economic trouble all over the world, because of the high costs, great number of cases and the high morbimortality among pregnant women. The International Federation of Contraception stimates that every year occurs around 5 millions of induced abortion; in Colombia in 1990 were reported 4.930 abortions during six month period. This is a retrospective-descriptive study, that was made in the Ginecology and Obstetrics Department of the Hospital San Vicente de Paúl University of Antioquia between october 1997 and march 1998, taking into account all pregnant women who sought medical attention between january $1^{\circ} 1994$ and december 31 1997. The most effected group of age was between 16-25 years, been most of them singles and house keepers. Most of them were multiparus, and the main complication was Septic Shock.

KEY WORDS: Septic abortion, pregnants, morbimortality, Hospital San Vicente de Paúl (HSVP).

El aborto séptico, definido como toda paciente embarazada con aborto completo, incompleto o frustro con temperatura superior a $37,8^{\circ} \mathrm{C}$ acompañado o no de secreción vaginal maloliente y dolor hip̀ogástrico (1), constituye en la actualidad un problema médico, social y económico a nivel mundial debido a la gxan demanda de casos, los elevados costos y las tasas de morbimortalidad materna. Según una estimación de 1991, en todo el mundo se provocaron 44 millones de abortos por año, de los cuales 15 millones fueron realizados clandestinamente (2); en Latinoamérica, la Federación Internacional de Planificación Familiar calcula que cada año ocurren 5 millones de abortos inducidos (3). Las cifras en Colombia no son menos escandalosas: en un estudio realizado durante 1990 en varios centros asistenciales del país, se informaron 4.930 abortos en un período de 6 meses; de estos, el $15,7 \%$ fueron clasificados como sépticos al

Internos. Universidad de Antioquia.

Profesor Departamento de Obstetricia y Ginecología.

Universidad de Antioquia. ingreso (4). El porcentaje de defunciones maternas por aborto séptico, de acuerdo con estudios realizados en el Instituto Materno-Infantil de Bogotá y el Hospital San Vicente de Paúl (HSVP) de Medellín durante el período de 1963-1968, osciló entre un $23 \%$ y $45 \%$, siendo la primera causa de muerte la sepsis; desafortunadamente estas cifras pueden ser aún mayores si tenemos en cuenta el carácter clandestino del aborto en nuestro país.

Las estadísticas indican que las mujeres con mayor nivel de instrucción residentes en las zonas urbanas recurren más al aborto, utilizando para ello los servicios de personal calificado desde el punto de vista médico; un fenómeno totalmente opuesto se observa en mujeres procedentes de la zona rural o con bajo nivel de instrucción (2). La población más frecuentemente afectada comprende mujeres solteras mayores de 20 años y casi siempre primigestantes (3).

En los países en donde el aborto está legalizado existen tres métodos para terminar la gestación:

1. La evacuación instrumental del útero

2. La inducción médica de las contracciones

3. La cirugía uterina 
Cuando el aborto es clandestino se utilizan maniobras diversas. En un estudio realizado en el HSVP (19681969), se observó que se emplearon muchos métodos abortivos, algunos de los cuales no causaron ningún efecto sobre el embarazo ni sobre la paciente, otros fueron eficaces para producir el aborto y aún para poner en peligro la salud materna. El método más utilizado en este estudio fue la sonda $(78,2 \%)$, y únicamente cuando hubo automaniobras se pudo constatar el empleo de otros métodos. En el Instituto Materno Infantil también es el método más frecuente $(51 \%)$. Entre los cuerpos extraños más usados se encuentran lápices y alambres. Actualmente se utiliza el tallo corto y desecado de laminaría, una alga feofícea, para provocar la dilatación del cuello e inducir el aborto, debido a la propiedad que tiene de hincharse en forma considerable cuando se pone en contacto con el agua.

Vale la pena señalar la importancia que está tomando el uso de drogas que modifican la contractilidad uterina (prostaglandinas, antiprogestágenos, etc.) entre las caulsas de aborto séptico, porque definitivamente hay que eliminar la idea de que aborto séptico es igual a maniobras abortivas; las anteriores drogas causan abortos que también tienen la posibilidad de sobreinfectarse y de convertirse en sépticos, y eso es lo que se está viendo en los centros hospitalarios con mayor frecuencia.

En lo referente a la microbiología, la infección es producida por la flora potencialmente patógena que se encuentra en la vagina y el endocérvix, constituida principalmente por anaerobios, enterobacilos, pseudomonas, bacteroides y clostridiums (6-7).

Estos gérmenes generalmente se encuentran asociados y actúan en el organismo ocasionando necrosis tisular y hemólisis. Para el tratamiento médico, hay un amplio espectro de antibióticos cuyo uso está definido por el grado clínico, para cuya determinación sigue siendo utilizada la clasificación de Newirth $(3,8)$ que va del grado I al III, siendo este último el más severo, requiriendo en algunas ocasiones tratamiento quirúrgico. Las complicaciones son de diversa gravedad y dependen de múltiples factores tales como: edad gestacional, lugar donde se realizó la maniobra abortiva, persona que ejecutó el procedimiento y método utilizado, entre otros.

Entre nosotros las estadísticas hospitalarias sobre aborto séptico son escasas. Los datos más recientes encontrados en el Hospital San Vicente de Paúl datan de 1971 (1), requiriéndose nuevos estudios que permitan esclarecer la frecuencia e importancia del problema, características de la población afectada y del evento estudiado.

\section{Materiales y métodos}

El presente estudio de tipo descriptivo retrospectivo, de corte transversal, se llevó a cabo en el Departamento de Obstetricia y Ginecología de la Facultad de Medicina de la Universidad de Antioquia y Hospital San Vicente de Paúl de Medellín, entre el $1^{\circ}$ de octubre de 1997 y el 10 de marzo de 1998.

La población de estudio la constituyeron el total de pacientes gestantes que consultaron por aborto séptico en el Servicio de Urgencias del Departamento de Obstetricia y Ginecología de dicha institución durante el período comprendido entre el $1^{\circ}$ de enero de 1994 y el 31 de diciembre de 1997.

Se definió que una paciente tenía un aborto séptico si al momento de la consulta cursaba con un aborto completo, incompleto o frustro con temperatura superior a $37,8^{\circ} \mathrm{C}$ acompañado o no de secreción vaginal maloliente y dolor hipogástrico. La clasificación clínica que se utilizó fue la de Newirth: $(3,8)$

Grado I: la infección localizada al útero. Grado II: infección diseminada a otras estructuras pélvicas, además del útero. Grado III: infección diseminada más allá de las estructuras pélvicas (asociada a septicemia, a peritonitis generalizada y a shock séptico).

La unidad de análisis para este estudio, la constituyen cada una de las historias clínicas de las pacientes con diagnóstico de aborto séptico.

Fueron tenidos en cuenta como criterios de exclusión: pacientes que no fueron registradas en el libro de ingresos del Servicio de Urgencias de este departamento, pacientes registradas con diagnóstico de aborto séptico que no tenían número de historia clínica, pacientes registradas erróneamente con diagnóstico de aborto séptico.

Finalmente, para la recolección de los datos se elaboró un formulario creado por los investigadores. Estos fueron obtenidos directamente de las historias clínicas de las pacientes con diagnóstico de aborto séptico, que se encontraban archivadas en el Servicio de Estadística del Hospital San Vicente de Paúl.

La información se registró en la Sala de Computadores del Departamento de Salud Pública de la Facultad de Medicina de la Universidad de Antioquia, donde también se analizaron los resultados, utilizando los programas: Epi info 6.0, Microsoft Word y Microsoft Excel.

\section{Resultados}

En el período comprendido entre el $1^{\circ}$ de enero de 1994 y el 31 de diciembre de 1997, se registraron en el servicio de Gineco-Obstetricia del Hospital San Vicente de Paúl, un total de 184 casos de pacientes con diagnóstico de aborto séptico. Se excluyen de este trabajo 27 casos. en los que no fue posible acceder a la historia clínica por diferentes razones.

De las 157 historias analizadas, el 58\% pertenecían a pacientes con edades comprendidas entre los 16 y 25 años; le sigue en frecuencia el grupo de edad comprendido entre los 26 y 35 años con un $33 \%$. La menor incidencia se presentó en el grupo de menores de 15 años. Se encontró que el $55 \%$ de las pacientes eran solteras, entre las cuales la causa de aborto más frecuente fue la inducida instrumentada con 39 casos; el 15\% eran casadas.

En cuanto a la escolaridad de las pacientes, en 120 casos $(76,4 \%)$, no se obtuvo este dato porque no se encontraban consignados en las historias clínicas de las pacientes; en los 37 casos restantes, $16(10,2 \%)$, correspondieron a secundaria incompleta.

El 53,5\% (84 casos) de las pacientes eran amas de casa, y $15(9,6 \%)$ eran estudiantes; sólo un $9,6 \%$ de las pacientes tenían un trabajo formal. Con respecto a las 
gravideces, ver tabla 1, cursaban su primer embarazo el $30,6 \%$ (48 casos) de las pacientes; el $69,4 \%$ eran multigestantes, de las cuales el $26,8 \%$ habían tenido 4 ó más embarazos.

Tabla 1

DISTRIBUCION POR GRAVIDECES

\begin{tabular}{|lcc|}
\hline Gravideces & Frecuencia & Porcentajes \\
\hline 1 & 48 & $30,6 \%$ \\
2 & 36 & $22,9 \%$ \\
3 & 30 & $19,1 \%$ \\
4 o más & 42 & $26,8 \%$ \\
Sin dato & 1 & $0,6 \%$ \\
\hline Total & 157 & $100,0 \%$ \\
\hline
\end{tabular}

Un total de 46 pacientes $(29,3 \%)$ informaron no tener partos previos y sólo 21 pacientes $(13,4 \%)$, tenían 4 ó más. Ver tabla 2.

Tabla 2

DISTRIBUCION POR PARIDAD

\begin{tabular}{|lcc|}
\hline Paridad & Frecuencia & Porcentaje \\
\hline 0 & 46 & $29,3 \%$ \\
1 & 42 & $26,8 \%$ \\
2 & 30 & $19,1 \%$ \\
3 & 15 & $9,6 \%$ \\
4 o más & 21 & $13,4 \%$ \\
Sin dato & 3 & $1,9 \%$ \\
\hline Total & 157 & $100,0 \%$ \\
\hline
\end{tabular}

Un total de 106 pacientes $(67, \%)$, no tenían abortos previos; $24 \%$ manifestaron haber tenido un aborto y el 6\% tenían 2 ó más abortos. Ver gráfica 1.

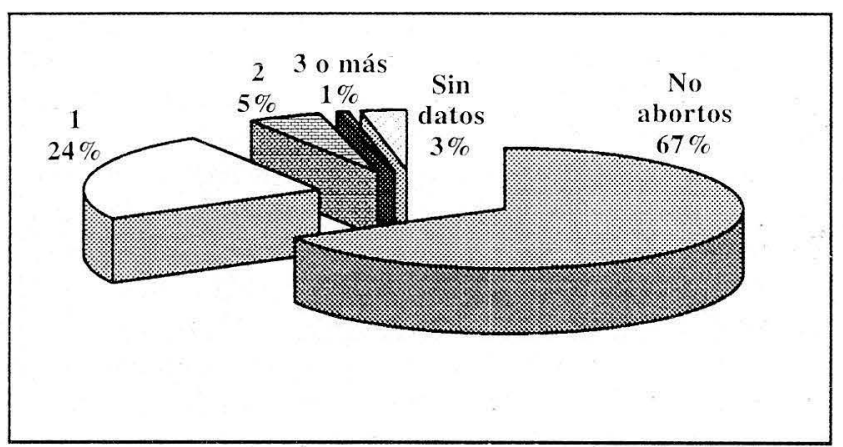

Gráfica 1

ABORTO SEPTICO - HUSVP

NUMERO DE ABORTOS PREVIOS
Del total de las pacientes, $35 \%$ tenían menos de 9 semanas de gestación; el $29,3 \%$ tenían entre 9 y 13 semanas y el $24,2 \%$ tenían entre 14 y 20 semanas. Ver gráfica 2 .

De las 157 pacientes, $53(34 \%)$ manifestaron maniobras abortivas con algún tipo de instrumentación, predominando en el grupo de edad de 16 a 25 años, y 52 (33\%) relataron inicio espontáneo de su cuadro, correspondiendo al grupo de 26 a 35 años; en el $11 \%$ la causa del aborto fue inducida no instrumentado. Ver gráfica 3.

El mayor número de abortos 113 casos (72\%), fueron clasificados clínicamente como aborto séptico I, y 25 casos $(15,9 \%)$ como aborto séptico III. Se presentaron 16 casos de aborto séptico II, (10,2\%). Ver tabla 3.

Tabla 3

DISTRIBUCION POR GRADO CLINICO

\begin{tabular}{|lcc|}
\hline Grado clínico & Frecuencia & Porcentaje \\
\hline Grado I & 113 & $72,0 \%$ \\
Grado II & 16 & $10,2 \%$ \\
Grado III & 25 & $15,9 \%$ \\
Sin dato & 3 & $1,9 \%$ \\
\hline Total & 157 & $100,0 \%$ \\
\hline
\end{tabular}

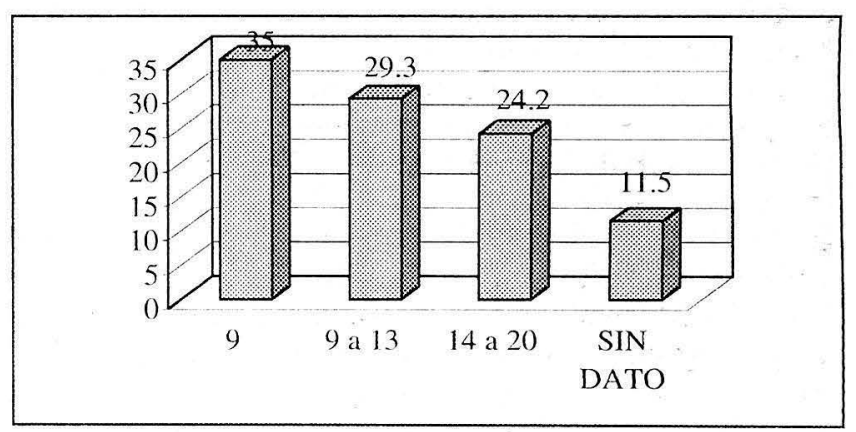

Gráfica 2

ABORTO SEPTICO - HUSVP

SEMANAS DE AMENORREA

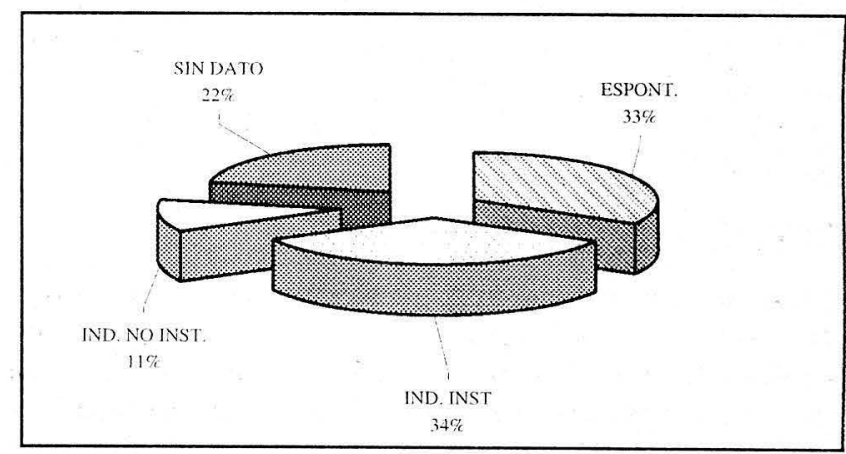

Gráfica 3

ABORTO SEPTICO - HUSVP

CAUSA DEL ABORTO 
En la gran mayoría de los casos $(52,8 \%)$, se utilizó penicilina - gentamicina como antibióticoterapia, seguida del biconjugado gentamicina-clindamicina en $31 \mathrm{ca}-$ sos $(19,7 \%)$. Ver tabla 4.

Tabla 4

DISTRIBUCION POR TRATAMIENTO MEDICO

\begin{tabular}{|lcr|}
\hline $\begin{array}{l}\text { Tratamiento } \\
\text { médico }\end{array}$ & Frecuencia & Porcentajes \\
\hline Peni + Genta & 83 & $52,8 \%$ \\
Gara + Genta & 31 & $19,7 \%$ \\
Peni + Genta + Metro & 16 & $10,2 \%$ \\
Peni + Genta + Clind & 9 & $5,7 \%$ \\
Otro esquema & 18 & $11,5 \%$ \\
\hline Total & 157 & $100,0 \%$ \\
\hline
\end{tabular}

A un total de 149 pacientes $(94,9 \%)$ se les realizó curetaje y sólo $7(4,5 \%)$ requirieron otro procedimiento adicional, siendo el más frecuente la anexohisterectomía $(3,2 \%)$. Ver tabla 5 .

Tabla 5

DISTRIBUCION POR TRATAMIENTO QUIRURGICO

\begin{tabular}{|lrr|}
\hline $\begin{array}{c}\text { Tratamiento } \\
\text { quirúrgico }\end{array}$ & Frecuencia & Porcentaje \\
\hline Curetaje & 149 & $94,9 \%$ \\
Histerectomía & 2 & $1,3 \%$ \\
Anexohisterectomía & 5 & $3,2 \%$ \\
Sin dato & 1 & $0,6 \%$ \\
Total & 157 & $100,0 \%$ \\
\hline
\end{tabular}

En cuanto a la estancia hospitalaria, 106 pacientes (79\%) permanecieron hospitalizadas de 2 a 6 días, mientras que sólo 18 pacientes ( $11 \%$ ) permanecieron menos de 1 día. El $89 \%$ de las pacientes no presentaron complicaciones; 18 pacientes se complicaron predominando el shock séptico en 8 casos (5\%), 7 de los cuales se presentaron en pacientes con edades entre 16 -35 años; le siguió en frecuencia la perforación uterina con $3 \%$. No se encontró ninguna complicación en las pacientes menores de 15 años.

Todas las pacientes menores de 15 años presentaron aborto séptico grado I; como aborto séptico grado III se encontraron 25 casos, de los cuales 24 estaban comprendidos entre los 16 y 35 años.

El aborto séptico grado I se presentó con mayor frecuencia en las primigestantes con 36 casos y el séptico III en las trigestantes con 10 casos.

El aborto inducido instrumentado se presentó con mayor frecuencia en las pacientes que habían tenido 30 menos gravideces con un total de 48 casos, y el aborto espontáneo se presentó con mayor frecuencia en las primigestantes y en las multigestantes (4 o más) con 18 y 16 casos, respectivamente. Ver gráfico 4.

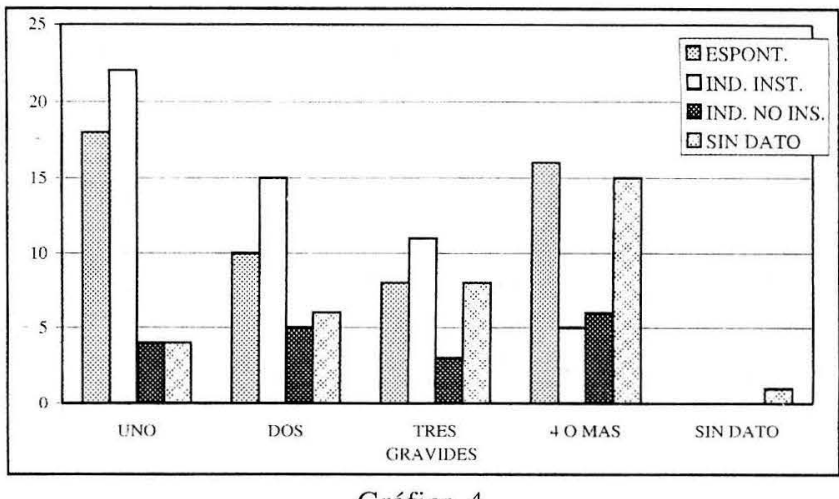

\section{ABORTO SEPTICO - HUSVP \\ CAUSA SEGUN GRAVIDECES}

Se encontró que de los 52 abortos espontáneos, 36 correspondían a pacientes sin abortos previos y de los 53 instrumentados, 43 pertenecían al mismo grupo. Ver gráfica 5 .

De las pacientes que no presentaron abortos previos, el $80 \%$ de los casos fueron clasificados como aborto séptico Grado I; en las pacientes que tenían uno o más abortos previos, este porcentaje disminuyó aunque siguió predominando sobre los otros grados clínicos. Ver gráfica $6(1)$.



Gráfica 5

ABORTO SEPTICO - HUSVP CAUSA SEGUN ABORTOS PREVIOS

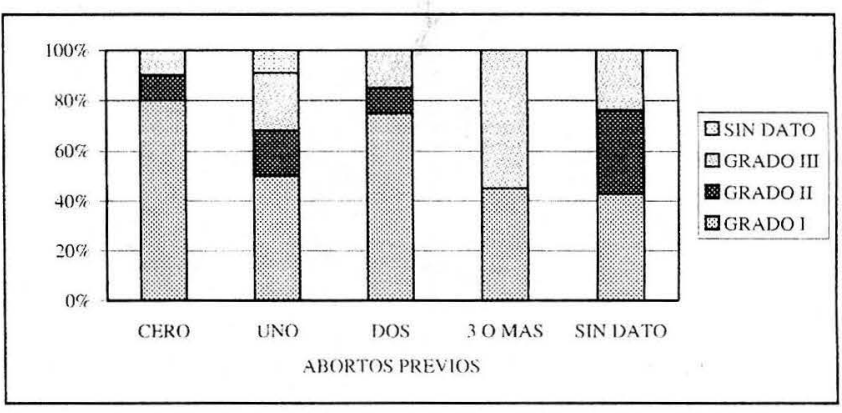

Gráfica 6

ABORTO SEPTICO - HUSVP

GRADO CLINICO SEGUN ABORTOS PREVIOS 
En los abortos con menos de 9 semanas de amenorrea, se encontró que el mayor número correspondía a abortos instrumentados, 20 de 52 casos; el aborto espontáneo se dio con más frecuencia entre las semanas 9 y 20, con 37 de 52 casos. Ver gráfica 7.

De los 113 casos de abortos sépticos I, 69\% se presentaron en pacientes con menos de 13 semanas de amenorrea, y 10 de $\operatorname{los} 25$ casos de séptico III, se encontraron entre las semanas 14 y 20 de amenorrea. Ver gráfica 8.

De las 157 pacientes 16 presentaron complicaciones; 8 casos correspondieron a shock séptico, de los cuales 4 se presentaron entre las 14 y 20 semanas de amenorrea. Ver gráfica 9 .

De las 157 pacientes, $113(72 \%)$ presentaron aborto séptico grado I. En 42 de estas pacientes (37\%) el aborto se presentó de forma espontánea; 48 tenían algún antecedente de maniobras abortivas y sólo 13 no fueron instrumentados. En 14 de los 25 sépticos III, la causa del aborto fue la instrumentación. Ver gráfica 10 .

Por otro lado, 139 del total de las pacientes no se complicaron. En 53 casos de abortos inducidos instrumentados se encontraron 7 complicaciones, distri-

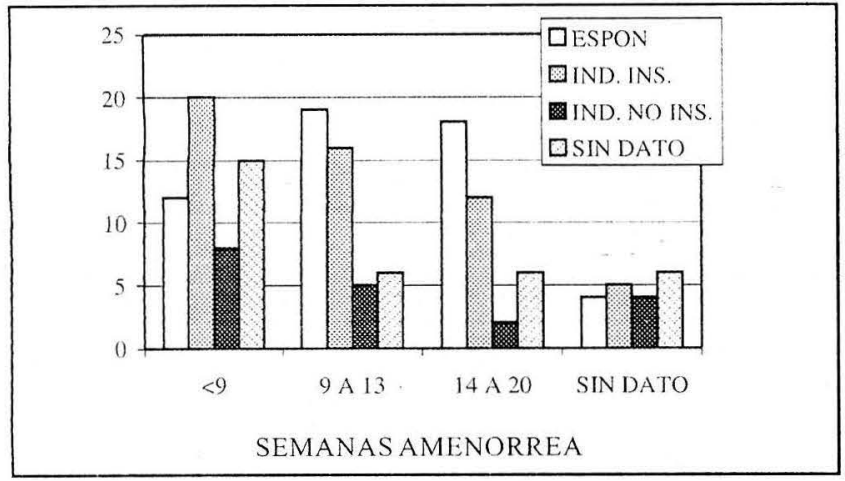

Gráfica 7

ABORTO SEPTICO - HUSVP SEMANAS DE AMENORREA SEGUN CAUSA

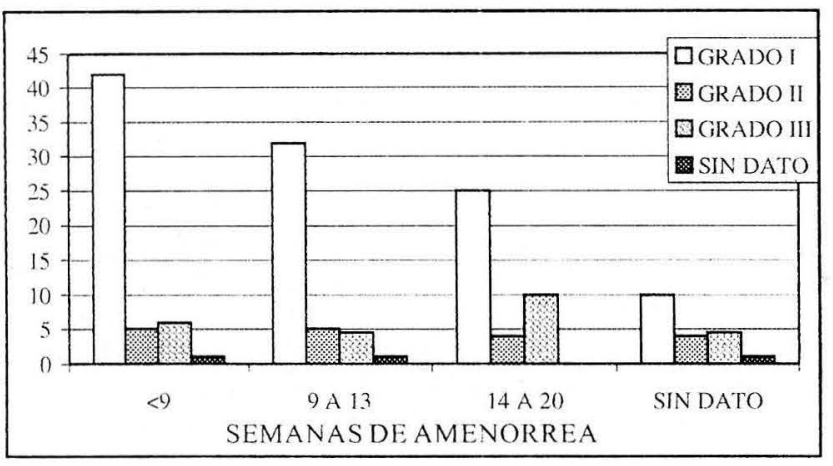

Gráfica 8

ABORTO SEPTICO - HUSVP

SEMANAS DE AMENORREA SEGUN

GRADO CLINICO buidos de la siguiente forma: 3 casos de shock séptico, 3 de perforación uterina y 1 muerte. De las pacientes con abortos inducidos no instrumentados 2 se complicaron, ambas con shock séptico, (ver gráfica 11), convirtiéndose este último en la principal complicación en cada uno de los 3 grados clínicos. Ver gráfica 12.

El $79 \%$ de las pacientes tuvo un tiempo de hospitalización entre 2 y 6 días; de ellas el 39\% y $43 \%$ presentaron aborto de causa espontánea o inducido, respectivamente.
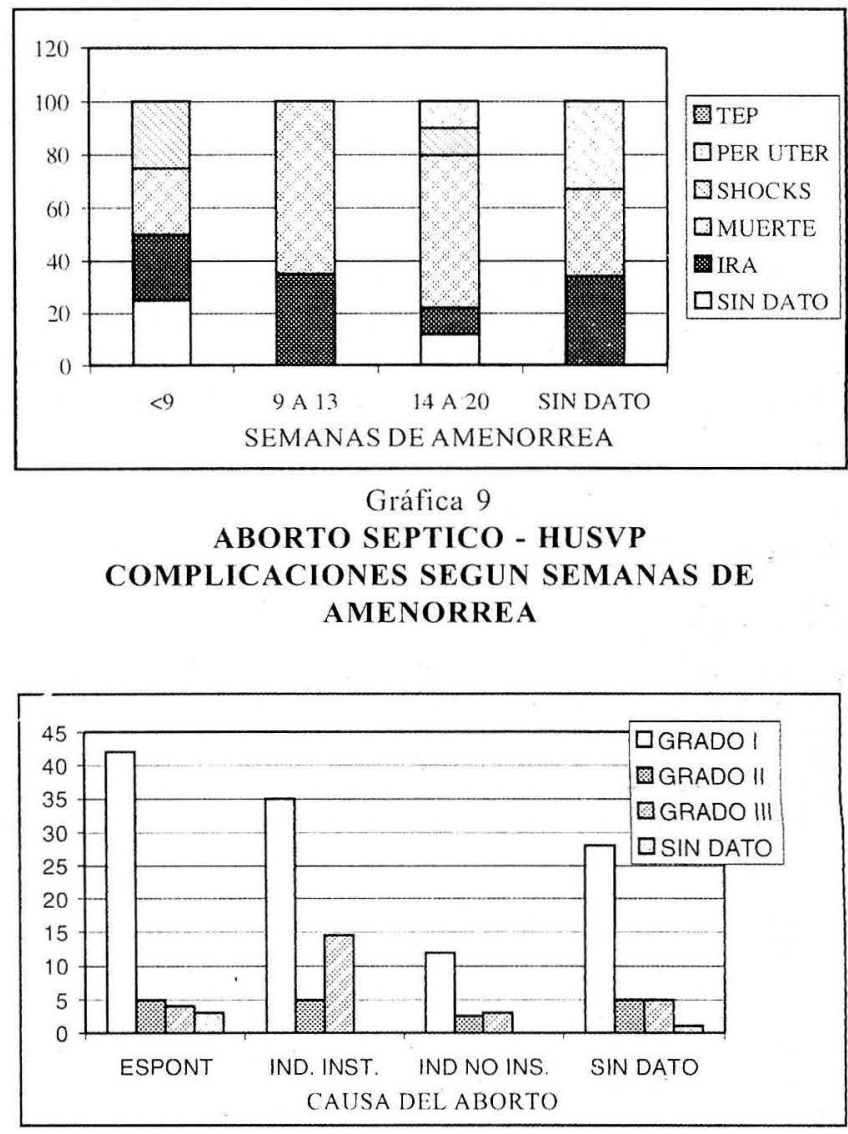

Gráfica 10

ABORTO SEPTICO - HUSVP GRADO CLINICO SEGUN CAUSA

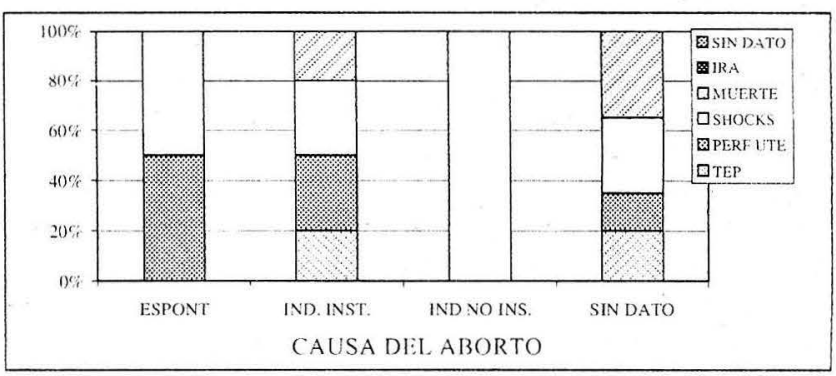

Gráfica 11

ABORTO SEPTICO - HUSVP

COMPLICACIONES SEGUN CAUSA DEL ABORTO 


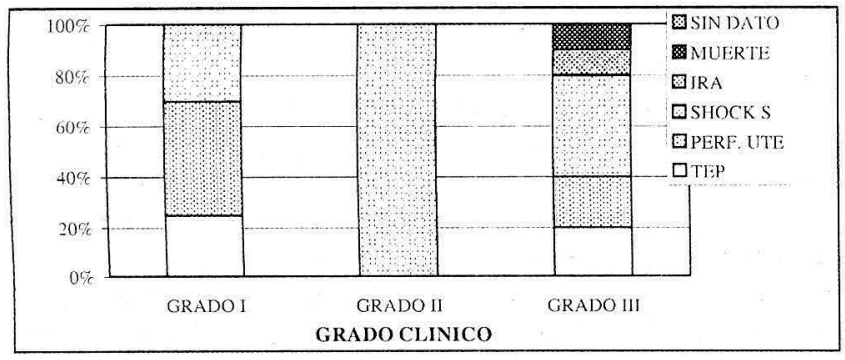

Gráfica 12

\section{ABORTO SEPTICO - HUSVP COMPLICACIONES SEGUN GRADO CLINICO}

Requirieron hospitalización mayor de 7 días sólo 14 pacientes $(9 \%)$, la mitad de ellas tenían aborto inducido instrumentado, y el $71 \%$ estaban clasificados como grado clínico III. Dieciocho de los casos (11\%) fueron ambulatorios, 8 de ellos espontáneos.

En cuanto al tratamiento, el procedimiento quirúrgico más utilizado en el manejo tanto de los abortos provocados como de los espontáneos, fue el curetaje con 149 (95\%) de los casos. Los 4 casos que requirieron anexohisterectomía fueron inducidos instrumentados. La histerectomía se practicó a un aborto espontáneo y a uno inducido no instrumentado.

Con respecto al tratamiento médico, el esquema antibiótico más utilizado fue el de Penicilina más gentamicina, independientemente del grado clínico y de la causa del aborto, con 83 pacientes, seguido del esquema gentamicina más clindamicina en 31 pacientes; en el aborto inducido instrumentado fue en donde más se utilizaron otros esquemas diferentes. Ver gráfica 13.

\section{Discusión}

La morbimortalidad del aborto séptico se ha reducido en los últimos años debido a los adelantos científicos, al mejor ambiente quirúrgico, a la gran gama de antibióticos de amplio espectro, a la disminución de instrumentación con fines abortivos y al aumento de recursos farmacológicos para abortar (10).

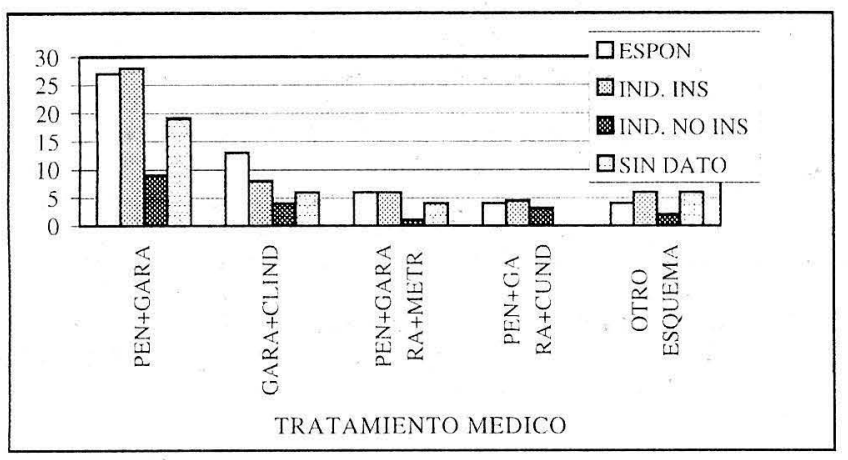

Gráfica 13
ABORTO SEPTICO - HUSVP
TRATAMIENTO MEDICO SEGUN CAUSA

Referente a la edad, se observa una tendencia a la disminución de la presentación del aborto séptico, al compararlo con otros estudios que muestran mayor incidencia en pacientes con edades comprendidas entre los 25 y 34 años $52 \%$ (6) y $42 \%$ (4); aunque en otro estudio publicado en 1974 se informaron cifras similares a las nuestras (10). Este fenómeno podría explicarse porque cada vez se presentan embarazos a edades más tempranas, con las implicaciones socioeconómicas que esto conlleva (madre-solterismo, rechazo en el hogar, deserción escolar, etc.); además de las complicaciones desde el punto de vista fisiológico inherentes a un embarazo a temprana edad (14). Lo anterior puede ser la explicación para que en el rango de edad entre 16 y 25 años la primera causa de aborto sea la instrumentada, contrario a lo que se observa en el grupo de mayores de 26 años en quienes predomina el aborto espontáneo.

Vemos como en el grupo de pacientes menores de 15 años, todas presentaron un aborto séptico grado I sin ningún tipo de complicación, probablemente por el escaso número de pacientes encontradas en este rango de edad: 5 pacientes.

En cuanto al estado civil, el aborto séptico continúa siendo más frecuente en las mujeres solteras, en relación con otro estudio realizado en 1966; (12) claro está que en dicho estudio el porcentaje de casadas fue superior al encontrado por nosotros. Sin embargo, Velasco encontró que el mayor porcentaje correspondía a mujeres casadas, $48 \%$ (11).

En lo referente a la ocupación, llama la atención cómo el grupo de desempleados constituye el $3,2 \%$ de la población, lo que estaría en contra de lo esperado, si tenemos en cuenta que una de las principales explicaciones para recurrir al aborto es la inestabilidad económica $(2,10)$.

Creemos que este bajo porcentaje podría ser explicado por la disparidad de conceptos al momento de elaborar la historia clínica, tomando como ama de casa a toda mujer que permanece en el hogar, sin especificar si ella es o no responsable del funcionamiento de éste; aparte de esto a muchas empleadas del servicio doméstico se les cataloga erróneamente como amas de casa.

El mayor porcentaje de abortos sépticos se presentó en pacientes multigestantes, dos o más embarazos, similar a lo informado en otros estudios donde el porcentaje de multigestantes oscilaba entre un $82 \%$ y $86 \%,(6,10$. 13) de las cuales entre el $43 \%$ y el $57 \%$ eran grandes multigestantes, 4 o más embarazos $(10,13)$.

Las multíparas presentan una mayor incidencia de maniobras abortivas, lo que se podría explicar por la afectación del presupuesto familiar con el advenimiento de otro hijo; además otra posible explicación sería el desconocimiento sobre métodos de planificación familiar, lo que contribuye a agravar el problema $(2,10)$.

En contraste con los datos encontrados en este estudio, Acosta y cols. hallaron que el $82 \%$ de las pacientes habían tenido abortos previos, de los cuales el $22 \%$ presentaron 2 o más abortos, y sólo el $18 \%$ no tenían antecedentes de abortos previos (10). Sin embargo otros autores informaron datos similares a los nuestros $(6,13)$. Al relacionar los abortos previos con la causa y el grado clínico no hay diferencia significativa. 
En cuanto a las semanas de amenorrea, en otra publicación se encontró un mayor porcentaje de abortos sépticos entre las semanas 9 y $12,51 \%$ (6). Por otro lado, Júbiz y col. informan una distribución similar de aborto séptico entre los grupos de pacientes que tenían menos de 9, de 9 a 12 y de 13 a 20 semanas de amenorrea (1).

En las pacientes con menos de nueve semanas de gestación predominó el aborto inducido instrumentado, datos que concuerdan con los hallados por Júbiz y cols., donde encuentran que en ese grupo de edad gestacional el $38 \%$ eran abortos provocados y el $23 \%$ eran espontáneos.

Pensamos que lo anterior está relacionado con las mayores facilidades técnicas de realizar el procedimiento a menor edad gestacional, lo cual es consistente con el mayor número de complicaciones y grado clínico más avanzado que se presentó en las pacientes que tenían entre 14 y 20 semanas de gestación; en contraste a lo anterior, Júbiz en su estudio no encontró significancia estadística entre el período de gestación y la clasificación clínica.

En lo referente a la causa del aborto, nuestros datos contrastan con los publicados por Acosta y cols., quienes informaron que un $67 \%$ de los abortos sépticos fueron espontáneos frente a un 33\% de provocados, de los cuales el $92 \%$ fueron instrumentados (10).

El alto porcentaje de maniobras abortivas instrumentadas con relación a las no instrumentadas, puede deberse al desconocimiento de nuevas modalidades abortivas, como el uso de fármacos por vía local o sistémica (2).

Al relacionar el grado clínico con la causa del aborto, los datos encontrados coinciden con lo esperado, ya que el mayor porcentaje de abortos sépticos grado III, tenían el antecedente de instrumentación. Además, en este mismo grupo se presentó el mayor número de complicaciones $(44,4 \%)$, siendo la más frecuente el shock séptico, lo que explica porque este grupo de pacientes recibió un tratamiento quirúrgico más agresivo y tuvo una estancia hospitalaria más prolongada (más de siete días).
En lo referente al grado clínico, los datos encontrados contrastan con lo informado por Júbiz y cols., donde no se encontró mayor diferencia entre los tres grados clínicos (grado I: $40 \%$, grado II: $35 \%$, grado III: $25 \%$ ) (1); otro autor publicó un predominio de los abortos sépticos grado III (48\%) (6). En cuanto al tratamiento médico se encontraron algunas discrepancias con respecto a los esquemas recomendados actualmente. Es así como el aborto séptico grado I, el esquema utilizado fue el biconjugado penicilina - gentamicina y en el grado III un $32 \%$ recibió este mismo tratamiento sin ninguna cobertura para gérmenes anaerobios gram negativos. Sin embargo de las 83 pacientes que recibieron este tratamiento, sólo se complicaron tres.

En lo que al tratamiento quirúrgico respecta, en un estudio realizado en el Instituto Materno Infantil (IMI) entre 1970 y 1971 , al $82,5 \%$ de las pacientes con aborto séptico grado I, se les realizó curetaje; al otro $17,5 \%$ además del anterior procedimiento se les practicó histerectomía abdominal: para nosotros estas cifras fueron del $98 \%$ y $2 \%$, respectivamente. En el estudio mencionado, al $37,5 \%$ de las pacientes con aborto séptico grado II se les practicó anexectomía y al $2,5 \%$ histerectomía.

En cuanto al aborto séptico grado III, al $58 \%$ se les realizó lavado y drenaje de cavidad abdominal; de éstas el $52,8 \%$ requirieron anexectomía y $34 \%$ histerectomía. Del $42 \%$ restante, al $9 \%$ se les realizó colpotomía, $13 \%$ histerectomía abdominal, anexectomía en el 3\%, curetaje sin necesidad de otro procedimiento al $17 \%$ (6).

Similar a lo informado en otras publicaciones, en la mayoría de las pacientes no se presentaron complicaciones $(1,6,10,13)$; éstas se presentaron en pacientes que estaban recibiendo otros esquemas de tratamiento diferentes a los propuestos en la tabla 4.

\section{BIBLIOGRAFIA}

1. Júbiz HA. Estudio sobre aborto séptico. HUSVP. Oct. 15, 1968 - oct. 14, 1969. Revista Colombiana de Obstetricia y Ginecología 1971; 22(4): 233-246

2. Hawker CS., Ferrando D. Aborto clandestino: una realidad latinoamericana. The Alan Guttmacher Institute. New York and Washington 1994.

3. Júbiz HA. Aborto. Actualización en Obstetricia y Ginecología/2. Edit. U. de A. Medellín, Colombia. 1994: 11-21.

4. Pardo F., Uriza G. Estudio sobre morbilidad y mortalidad por aborto en 36 instituciones de Bolivia, Colombia. Perú y Venezuela. Revista Colombiana de Obstetricia y Ginecología. 1991; 42(4): 287-297.

5. OMS. Complicaciones del aborto. 1995; 13-18.

6. Lozano A., Peralta MT. Morbi-mortalidad, clasificación y tratamiento del aborto séptico. Revista Colombiana de Obstetricia y Ginecología. 1981; 32(2): 104-134.

7. Jawest E., Melrick JL. Microbiología médica. Edit. Manual Moderno. 1996. $15^{\text {a }}$ edición
8. Botero J., Júbiz A. Obstetricia y Ginecología. Texto integrado. $5^{\text {a }}$ edición. Pág. 146-149.

9. Arteaga DC., Lozano A. Tratamiento del aborto séptico y la amnionitis con Sulbactan/Ampicilina. Revista Colombiana de Obstetricia y Ginecología. 1991; 42(2): 134-136.

10. Cantillo J., Rojas L. El aborto séptico y su aspecto económico social. Revista Colombiana de Ginecología y Obstetricia. 1974; 25(6): 409-417

11. Velasco G. Aborto séptico en el ICSS. Revista Colombiana de Obstetricia y Ginecología. 1975; 26(6): 341-352.

12. Santamaría PL. Aborto séptico y maniobras abortivas. Revista Colombiana de Obstetricia y Ginecología. 1966; 17(4): 272-290.

13. Navarro H. Aborto séptico. Revista Colombiana de Obstetricia y Ginecología. 1985; 36(4): 255-261.

14. Duarte A. ¿El embarazo en adolescentes es siempre de alto riesgo? Revista Colombiana de Obstetricia y Ginecología. 1975; 26(6) 385-395. 